\title{
Independence of the Residual Quadratic Sums in the Dispersion Equation with Noncentral $\chi^{2}$-Distribution
}

\author{
Nikolay I. Sidnyaev, Kristina S. Andreytseva \\ Bauman Moscow State Technical University, Moscow, Russia \\ E-mail: sidnyaev@yandex.ru,9259988800@mail.ru \\ Received May 6, 2011; revised July 1, 2011; accepted July 8, 2011
}

\begin{abstract}
A model adequacy test should be carried out on the basis of accurate aprioristic ideas about a class of adequate models, as in solving of practical problems this class is final. In article, the quadratic sums entering into the equation of the dispersive analysis are considered and their independence is proved. Necessary and sufficient conditions of existence of adequate models are resulted. It is shown that the class of adequate models is infinite.
\end{abstract}

Keywords: Noncentral $\chi^{2}$-Distribution, Dispersion Analysis, Adequate Models, Quadratic Sums

\section{Introduction}

The dispersive analysis is defined as the statistical method intended for estimation of influence of various factors on a result of experiment, so application area of this method becomes much wider. Unbiased estimate for unknown parameters is the sum of squares. The main idea of the dispersive analysis consists in splitting of this sum of squares of deviations into some components, each of which corresponds to the prospective reason of averages changing.

Let's consider decomposition of the residual sum of squares

$$
Q_{0}=Q_{1}+Q_{2}
$$

and we will prove independence of the summands $Q_{1}$ and $Q_{2}$. Two theorems and four auxiliary lemmas will be necessary for the proof.

\section{Preliminaries}

Lemma 2.1. The rank of composition of two matrixes $A$ and $B$ is less or equal to minimal rank of matrixes $A$ and Bi.e.

$$
r(A B) \leq \min (r(A), r(B)) .
$$

Proof. By a rule of matrixes multiplication, columns of matrix $A B$ are a linear combination of columns of a matrix $A$, then the number of linearly independent columns in $A B$ can't surpass the number of linearly inde- pendent columns in $A$; consequently

$$
r(A B) \leq r(A) .
$$

Doing similar reasoning for the lines (the lines of $A B$ are a linear combination of the lines $B)$, we will receive that $r(A B) \leq r(B)$. The lemma is proved.

Consequence of the inertia law of square-law forms (about quantity of invariants): if $Q=x^{\prime} A x$ is the square-law form with $n$ variables $x_{1}, \cdots x_{n}$ and its rank is equal to $r, r(A)=r$, then $r$ linear combinations of variables $x_{1}, \cdots x_{n}$ exist, for example, $z_{1}, \cdots, z_{r}$ such that $Q=\sum_{i=1}^{r} \lambda_{i} z_{i}^{2}$ and every $\lambda_{i}=1$ or -1 .

We will use the Kohran theorem as a simple consequence of the following theorem.

Theorem 2.1. Let $\sum_{i=1}^{N} y_{i}^{2}=Q_{1}+\cdots+Q_{s}$, where $Q_{j}$, $j=\overline{1, s}$, are the square-law form with rank $n_{j}$ from variables $y_{1}, \cdots, y_{N}$. Then the condition $n_{1}+n_{2}+\cdots+n_{s}=N$ is a necessary and sufficient condition for existence of the orthogonal transformation $z=A y$ translating a vector $y=\left(y_{1}, \cdots, y_{N}\right)^{\prime}$ into a vector $z=\left(z_{1}, \cdots, z_{N}\right)^{\prime}$ in such way, that

$$
Q_{1}=\sum_{i=1}^{n_{1}} z_{i}^{2}, Q_{2}=\sum_{i=n_{1}+1}^{n_{1}+n_{2}} z_{i}^{2}, \cdots, Q_{s}=\sum_{i=n_{1}+\ldots+n_{s-1}+1}^{n_{1}+\ldots+n_{s}} z_{i}^{2},
$$

Prove. Necessity.

If such orthogonal transformation exists, then 
$\sum_{i=1}^{N} y_{i}^{2}=\sum_{i=1}^{n_{1}+\cdots+n_{s}} z_{i}^{2}$. The left part is the square-law form of a rank $N$, and the right part is the square-law form of a rank $n_{1}+n_{2}+\cdots+n_{s}$. By the lemma 2.2, ranks of square-law forms are equal, i.e. $n_{1}+n_{2}+\cdots+n_{s}=N$.

\section{Sufficiency.}

As the rank $Q_{j}$ is equal $n_{j}$, then from a consequence of the inertia law of square-law forms, it follows that $n_{j}$ linear combinations $z_{1}, \cdots, z_{n_{j}}$ of variables $y_{1}, \cdots, y_{N}$ exist, such that $Q_{j}=\sum_{i} \lambda_{i} z_{i}^{2}$ where each $\lambda_{i}=1$ or -1 . For $Q_{1}$ indexes $i$ have values $1,2, \cdots, n_{1}$; for $Q_{2}-n_{1}+1, \cdots, n_{1}+n_{2}$ etc. Now, if $\sum_{i=1}^{s} n_{i}=N$, then $N$ linear combinations $z_{1}, \cdots, z_{N}$ exist, which in matrix designations can be written so: $z=A y$.

Using a diagonal matrix $D_{N \times N}$ with diagonal elements $\lambda_{1}, \cdots, \lambda_{N}$, we receive that

$$
\sum_{j=1}^{s} Q_{j}=\sum_{i=1}^{N} \lambda_{i} z_{i}^{2}=z^{\prime} D z=y^{\prime} A^{\prime} D A y .
$$

On the other hand $\sum_{j=1}^{s} Q_{j}=\sum_{i=1}^{N} y_{i}^{2}=y^{\prime} y$. As the symmetric matrix of the square-law form is unique, it is concluded that $A^{\prime} D A=I$, hence, it is nondegenerate. Now we will prove that $D=I$. Let $\lambda_{k}=-1$. Then under the formula $y=A^{-1} z$ we can find the values of $y_{1}, \cdots, y_{N}$ corresponding to values $z_{i}=0$ at $i \neq k$ and $z_{k}=1$, and for these values

$$
\sum_{i=1}^{N} y_{i}^{2}=\sum_{i=1}^{N} \lambda_{i} z_{i}^{2}=\lambda_{k}=-1
$$

that is impossible. Hence, $D=I$ and $A^{\prime} A=I$. Last equality shows that transformation $z=A y$ is orthogonal. The theorem is proved.

Remark. The condition $\sum_{i=1}^{s} n_{i}=N$ makes the squarelaw forms $Q_{i}$ positive definite, as at orthogonal transformation, it turns out that all their characteristic numbers are equal 0 or 1 .

Theorem 2.2 [1]. Let random variables $y_{i}, i=\overline{1, N}$, are independent and have normal distributions $N\left(\eta_{i}, 1\right)$ accordingly. Let further

$$
\sum_{i=1}^{N} y_{i}^{2}=Q_{1}+\cdots+Q_{s}
$$

where $Q_{i}, i=\overline{1, s}$, - the square-law form from variables $y_{1}, \cdots, y_{N}$ of $n_{i}$ rank. Then $Q_{1}, \cdots, Q_{s}$ have independent noncentral $\chi^{2}$-distribution with $n_{1}, \cdots, n_{s}$ freedom degrees accordingly, in only case, when $\sum_{i=1}^{s} n_{i}=N$. If $\delta_{i}$ is the parameter of noncentrality $Q_{i}$, then the value $\delta_{i}^{2}$ can be received by replacement $y_{j}$ on $Q_{i}$, i.e. if $Q_{i}=y^{\prime} A_{i} y$, then $\delta_{i}^{2}=\eta^{\prime} A_{i} \eta$, where $\eta=\left(\eta_{1}, \cdots, \eta_{N}\right)^{\prime} ; y=\left(y_{1}, \cdots, y_{N}\right)^{\prime}$.

Proof. Necessity.

If $Q_{1}, \cdots, Q_{s}$ are the independent random variables with $\chi^{2}$-distribution with $n_{1}, \cdots, n_{s}$ freedom degrees accordingly, then $\sum_{j=1}^{s} Q_{j}$ has noncentral $\chi^{2}$-distribution with $\sum_{j=1}^{s} n_{j}$ freedom degrees. As $\sum_{i=1}^{N} y_{i}^{2}$ has noncentral $\chi^{2}$-distribution with $N$ freedom degrees, and $\sum_{i=1}^{N} y_{i}^{2}=\sum_{j=1}^{s} Q_{j}$, hence, $\sum_{j=1}^{s} n_{j}=N$.

Sufficiency. Let $\sum_{j=1}^{s} n_{j}=N$. Then at orthogonal transformation $z=A y \quad$ (theorem 2.1), random variables $z_{1}, \cdots, z_{N}$ will be independent and normally distributed. From parities (2.1) and definitions of noncentral $\chi^{2}$ distributions follows that $Q_{1}, \cdots, Q_{s}$ have independent noncentral $\chi^{2}$-distributions with $n_{1}, \cdots, n_{s}$ freedom degrees accordingly. The theorem is proved.

\section{Auxiliary Theorems and Lemmas}

We will assume that the space of values of random variables is split into finite $r$ parts $s_{1}, \cdots, s_{n}$ without the general points, and let $p_{1}, \cdots, p_{n}$-probabilities $P_{i}=P\left\{X \in S_{i}\right\}, \quad \sum P_{i}=1$.

Let's assume that all $p_{i}>0$. Let $v_{i}$ is the number of observed values of random variables $-X$ belongs to set $s_{i}$.

Let's consider a vector $\left(v_{1}, \cdots, v_{r}\right)$. As a divergence measure between empirical and theoretical distribution we will consider $\sum_{i=1}^{r} c_{i}\left(\frac{v_{i}}{n}-p_{i}\right)^{2}$, where factors $c_{i}$ could be chosen random. Pearson has shown $([2,3])$ that if $c_{i}=\frac{n}{p_{i}}$, then received measure

$$
\chi_{n}^{2}=\sum_{1}^{r} \frac{n}{p_{i}}\left(\frac{v_{i}}{n}-p_{i}\right)^{2}=\sum_{1}^{r} \frac{v_{i}^{2}}{n p_{i}}-n
$$

possesses extremely simple properties.

Theorem 3.1. At $n \rightarrow \infty$, distribution $\chi_{n}^{2}$ aspires to distribution $\chi^{2}$ with $r-1$ degrees of freedom.

On the basis of this theorem by the set significance value $\alpha$ we will find the number $\chi_{n}^{2}$ from the condition 


$$
P\left\{\chi^{2}>\chi_{\alpha}^{2}\right\}=\alpha
$$

The hypothesis $H_{0}$ is rejected, if $\chi_{n}^{2}>\chi_{\alpha}^{2}$.

At the proof of the theorem the following lemma is required to us.

Lemma 3.1. Let $v_{1}, \cdots, v_{r}$ - the whole non-negative numbers, and $v_{1}+v_{2}+\cdots+v_{r}=n$. Number of ways, by means of which $n$ elements can be divided into $r$ groups, the first of which contains $v_{1}$ elements, the second elements $-v_{2}, \cdots, r_{i}-v_{r}$ elements, is equal to

$$
\frac{n !}{v_{1} ! \cdots v_{r} !} \text {. }
$$

Proof. The first group of $v_{1}$ elements can be chosen by $C_{n}^{v_{1}}$ ways. After the first group is formed, $n-v_{1}$ elements remain. Therefore, the second group of $v_{2}$ elements can be chosen by $C_{n-\nu_{1}}^{v_{2}}$ ways etc. After formation of $r-1$ groups, $n-v_{1}-\cdots v_{r-1}=v_{r}$ elements remain, which form the last group. Thus, the number of all possible ways by means of which $n$ elements can be distributed on $r$ groups, from which the first contains $v_{1}$ elements, $\cdots, r_{i}$ contains $v_{r}$ elements, is equal to

$$
C_{n}^{v_{1}} \cdot C_{n-v_{1}}^{v_{2}} \cdots C_{n-v_{1}-\cdots-v_{r-2}}^{v_{r-1}} \cdot
$$

Using the formula $C_{n}^{k}=\frac{n !}{k !(n-k) !}$, we will receive the lemma statement.

Proof. Result of any test with probability
$P_{i}=P\left\{X \in S_{i}\right\} \quad$ will belong to set $S_{i}$. Therefore, on the basis of a lemma 2.1 , the probability of that $v_{1}$ values will belong to set $S_{1}, \cdots, v_{r}$ values will belong to set $S_{r}$, is equal to

$$
\frac{n !}{v_{1} ! v_{2} ! \cdots v_{r} !} P_{1}^{v_{1}} \cdots P_{r}^{v_{r}}
$$

This expression, as it is easy to see, is the general member of decomposition $\left(P_{1}+\cdots+P_{r}\right)^{n}$. Joint distribution of a random vector $v=\left(v_{1}, \cdots, v_{r}\right)$ is set by expession (3.3) and is polynominal distribution. We will find the characteristic function with polynominal distributions. We have

$$
\begin{aligned}
M e^{i(t, v)} & =M \mathrm{e}^{i t_{1} v_{1}} \cdots \mathrm{e}^{i t_{r} v_{r}} \\
& =\sum_{\substack{v_{i} \neq 0 \\
v_{1}+\cdots+v_{r}=n}} \mathrm{e}^{i t_{1} v_{1}} \cdots \mathrm{e}^{i t_{r} v_{r}} \frac{n !}{v_{1} ! \cdots v_{r} !} P_{1}^{v_{1}} \cdots P_{r}^{v_{r}} \\
& =\left(P_{1} \mathrm{e}^{i t_{1}}+\cdots+P_{r} \mathrm{e}^{i t_{r}}\right)^{n} .
\end{aligned}
$$

Let's enter new quantities:

$$
x_{i}=\frac{v_{i}-n p_{i}}{\sqrt{n p_{i}}}, \quad i=1,2, \cdots, r .
$$

Then obviously, $\sum x_{i} \sqrt{p_{i}}=0, \chi_{r}^{2}=\sum_{1}^{r} x_{i}^{2}$. We will find characteristic function of a random vector $x=\left(x_{1}, \cdots, x_{r}\right)$. We have

$$
\begin{aligned}
\varphi\left(t_{1}, \cdots, t_{r}\right) & =M \mathrm{e}^{i\left(t_{1} x\right)}=M \mathrm{e}^{i\left(t, \frac{v-n p}{\sqrt{n p}}\right)}=\sum_{\substack{v_{i} \geq 0 \\
\sum v_{i}=n}} \mathrm{e}^{i t_{1} \frac{v_{1}-n p_{1}}{\sqrt{n p_{1}}}} \cdots \mathrm{e}^{i t_{r} \frac{v_{r}-n p_{r}}{\sqrt{n p_{r}}}} \cdot \frac{n !}{v_{1} ! \cdots v_{r} !} P_{1}^{\nu_{1}} \cdots P_{r}^{v_{r}} \\
& =\mathrm{e}^{-i \sum t_{k} \frac{n p_{k}}{\sqrt{n p_{k}}}} \cdot \sum_{\sum_{i_{i} \geq 0} v_{i}=n} \mathrm{e}^{i t_{1} \frac{v_{1}}{\sqrt{n p_{1}}}} \cdots \mathrm{e}^{i t_{r} \frac{v_{r}}{\sqrt{n p_{r}}}} \cdot \frac{n !}{v_{1} ! \cdots v_{r} !} P_{1}^{\nu_{1}} \cdots P_{r}^{v_{r}}=\mathrm{e}^{-i \sqrt{n} \sum t_{k} \sqrt{p k}}\left(P_{1} \mathrm{e}^{\frac{i t_{1}}{\sqrt{n p_{1}}}}+\cdots+P_{r} \mathrm{e}^{\frac{i t_{r}}{\sqrt{n p_{r}}}}\right)
\end{aligned}
$$

Further, for any fixed $t_{1}, \cdots, t_{r}^{n}$, we will receive

$$
\ln \varphi\left(t_{1}, \cdots, t_{r}\right)=n \ln \left(\sum P_{k} \mathrm{e}^{i t_{k} / \sqrt{n p_{k}}}\right)-i \sqrt{n} \sum t_{k} \sqrt{p_{k}}
$$

From decompositions $\mathrm{e}^{x}=1+x+\frac{x^{2}}{2 !}+O\left(x^{3}\right), \ln (1+x)=x-\frac{x^{2}}{2}+\frac{1}{3} R,|R| \leq\left|x^{3}\right|$, and from (2.5) follows that

$$
\begin{aligned}
& \sum p_{k} \mathrm{e}^{\frac{i t_{k}}{\sqrt{n p_{k}}}}=\sum p_{k}+\sum p_{k}^{\frac{i t_{k}}{\sqrt{n p_{k}}}}+\frac{1}{2} \sum p_{k} \frac{(i)^{2} t_{k}^{2}}{\left(\sqrt{n p_{k}}\right)^{2}}+O\left(n^{-3 / 2}\right)=\sum p_{k}+\sum p_{k}^{\frac{i t_{k}}{\sqrt{n p_{k}}}}-\frac{1}{2} \sum \frac{t_{k}^{2}}{n}+O\left(n^{-3 / 2}\right), \\
& \ln \varphi\left(t_{1}, \cdots, t_{r}\right)=n \ln \left[1+\frac{i}{\sqrt{n}} \sum t_{k} \sqrt{p_{k}}-\frac{1}{2 n} \sum t_{k}^{2}+O\left(n^{-3 / 2}\right)\right]-i \sqrt{n} \sum t_{k} \sqrt{p_{k}} \\
& =n \ln \left[\frac{i}{\sqrt{n}} \sum t_{k} \sqrt{p_{k}}-\frac{1}{2 n} \sum t_{k}^{2}+O\left(n^{-3 / 2}\right)\right]-\frac{n}{2}\left[-\frac{i}{\sqrt{n}} \sum t_{k} \sqrt{p_{k}}-\frac{1}{2 n} \sum t_{k}^{2}+O\left(n^{-3 / 2}\right)\right]^{2}+\frac{n}{3} R-i \sqrt{n} \sum t_{k} \sqrt{p_{k}} \\
& =-\frac{1}{2} \sum t_{k}^{2}+\frac{1}{2}\left(\sum t_{k} \sqrt{p_{k}}\right)^{2}+O\left(n^{-1 / 2}\right)
\end{aligned}
$$


So, now we can receive that

$$
\lim _{n \rightarrow \infty} \varphi\left(t_{1}, \cdots, t_{r}\right)=\mathrm{e}^{-\frac{1}{2}\left[\sum t_{k}^{2}-\left(\sum t_{k} \sqrt{p_{k}}\right)^{2}\right]}=\mathrm{e}^{-\frac{1}{2} Q\left(t_{1}, \cdots, t_{r}\right)}
$$

The square-law form

$$
Q\left(t_{1}, \cdots, t_{r}\right)=\sum t_{k}^{2}-\left(\sum t_{k} \sqrt{p_{k}}\right)^{2}
$$

has a matrix $\Lambda=I-p p^{\prime}$ where I designates an individual matrix, and $P$ is a vector-column, replacing $t_{1}, \cdots, t_{r}$ with new variables $u_{1}, \cdots, u_{r}$ by means of orthogonal transformation, at which $u_{r}=\sum t_{k} \sqrt{p_{k}}$, we will receive

$$
Q\left(t_{1}, \cdots, t_{r}\right)=\sum_{1}^{r} t_{k}^{2}-\left(\sum_{1}^{r} t_{k}^{2} \sqrt{p_{k}}\right)^{2}=\sum_{1}^{r} u_{k}^{2}-u_{r}^{2}=\sum_{1}^{r-1} u_{r}^{2} .
$$

So, the square-law form $Q\left(t_{1}, \cdots, t_{r}\right)$ is non-negative and also has a rank $r-1$, i.e. at $n \rightarrow \infty$, joint characteristic function of quantities $x_{1}, \cdots, x_{r}$ aspires to the expression $\exp (-1 / 2 Q)$, which is characteristic function of some nonintrinsic normal distribution of a rank $r-1$, in which all weight is concentrated to a hyperplane $\sum x_{k} \sqrt{p_{k}}=0$.

From the continuity theorem follows that $x_{1}, \cdots, x_{r}$ have nonintrinsic normal distribution with zero average and a matrix of the second moments $\Lambda$. From here we receive that the quantity $\chi_{r}^{2}=\sum x_{k}^{2}$ in a limit has distribution $\chi^{2}$ with $r-1$ freedom degrees.

\section{Noncentral $\chi^{2}$-Distribution}

Let's consider that $y_{1}, y_{2}, \cdots, y_{n}$-the independent random variables with normal distribution with an average $\mu_{i} \quad(i=1,2, \cdots, n)$ and a dispersion 1, i.e. $y_{i} \sim N$ $\left(\mu_{i}, 1\right)(i=1,2, \cdots, n)$. Then random variable distribution

$$
u=\sum_{i=1}^{n} y_{i}^{2}
$$

is called as noncentral $\chi^{2}$-distribution [1-3].

The quantity $\sqrt{u}$ represents radius of a hypersphere in n-dimensional space $[1,4]$.

Random variable distribution $u$ depends only on parameters $\mathrm{n}$ and $\sigma=\left(\sum_{i=1}^{n} \mu_{i}^{2}\right)^{1 / 2}$. Therefore it also names as noncentral $\chi^{2}$-distribution with $\mathrm{n}$ degrees of freedom and non-centrality parameter $\sigma[2,5]$. In this case, following [4], a random variable $\mathrm{u}$ we will designate

$$
u=\chi_{n ; \sigma}^{2}
$$

If, $\sigma=0$, i.e. $\mu_{i}=0 \quad(i=1,2, \cdots, n)$, distribution of random variable u named as central $\chi^{2}$-distribution or it is simple $\chi^{2}$-distribution with $n$ degrees of freedom and a random variable $u$ we will designate

$$
u=\chi_{n}^{2} \text {. }
$$

Let $\left.P\left\{\chi_{n}^{2}\right\rangle \chi_{\alpha ; \sigma}^{2}\right\}=\alpha$. Quantity $\left.\chi_{\alpha ; n}^{2}\right\rangle 0$ is named as a threshold or $\alpha$-percentage point of $\chi^{2}$-distribution with $\mathrm{n}$ freedom degrees. Its values for various $\alpha$ and $\mathrm{n}$ [5]. The mean and variance of a random variable $\chi_{n ; \sigma}^{2}$ are

$$
M\left\{\chi_{n ; \sigma}^{2}\right\}=n+\sigma^{2} ; D\left\{\chi_{n ; \sigma}^{2}\right\}=2 n+4 \sigma^{2} .
$$

If $u_{1}=\chi_{n_{1} ; \sigma_{1}}^{2}$ and $u_{2}=\chi_{n_{2} ; \sigma_{2}}^{2}$-independent random variables, then from definition of noncentral $\chi^{2}$-distribution it follows that their sum $u=u_{1}+u_{2}=\chi_{n ; \sigma}^{2}$ has noncentral $\chi^{2}$-distribution with $n=n_{1}+n_{2}$ degrees of freedom and parameters of not centrality $\sigma=\left(\sigma_{1}^{2}+\sigma_{2}^{2}\right)^{1 / 2}$.

\section{Main Results}

For the proof of $Q_{1}$ and $Q_{2}$ independence we will result following auxiliary statements.

Lemma 5.1. The rank of the sum of square-law forms doesn't surpass the sum of their ranks.

Proof. It is enough to show that if $A_{1}$ and $A_{2}$ are matrixes of one order and the rank $A_{i}$ is equal to $n_{i}$, then $r\left(A_{1}+A_{2}\right) \leq r_{1}+r_{2}$. For the vector space generated by columns $A_{i}$, we will choose basis from vectors $r_{i}$. As columns $A_{1}+A_{2}$ are equal to the sums of corresponding columns $A_{1}$ and $A_{2}$, then they are linear combinations $r_{1}+r_{2}$ of vectors of two bases; hence, the number of linearly independent columns in $A_{1}+A_{2}$ can't surpass $r_{1}+r_{2}$. Hence, $r\left(A_{1}+A_{2}\right) \leq r_{1}+r_{2}$. The lemma is proved.

Consequence. If $\sum_{i=1}^{N} y_{i}^{2}=Q_{1}+\cdots+Q_{s}$, where the rank $Q_{j}$ is less or equal to $n_{j}, j=\overline{1, s}$ and if $n_{1}+n_{2}+\cdots+n_{s}=N$, then $r\left(Q_{j}\right)=n_{j}, j=\overline{1, s}$.

Proof. It follows directly from a lemma 5.1. On the one hand

$$
r\left(\sum_{j=1}^{s} Q_{j}\right) \leq \sum_{j=1}^{s} r\left(Q_{j}\right) \leq \sum_{j=1}^{s} n_{j}=N
$$

and on the other hand

$$
r\left(\sum_{j=1}^{s} Q_{j}\right)=r\left(\sum_{i=1}^{N} y_{i}^{2}\right)=N .
$$

Hence,

$$
\sum_{j=1}^{s} r\left(Q_{j}\right)=N .
$$

Under a condition of $r\left(Q_{j}\right) \leq n_{j}, j=\overline{1, s}$, performance of last equality is possible only when $r\left(Q_{j}\right)=n_{j}$, 
$j=\overline{1, s}$, as proves a consequence.

Lemma 5.2. If $Q$ is the square-law form from variables $y_{1}, \cdots, y_{N}$ and can be expressed as the square-law form from the variables $z_{1}, \cdots, z_{p}$ which are linear combinations of $y_{1}, \cdots, y_{N}$, то $r(Q) \leq p$.

Proof. Let $Q=y^{\prime} A_{N \times N} y=z^{\prime} B_{p \times p} z$ and $z=C_{p \times N} y$, $A$ and $B$ are symmetric. Then from equality $Q=y^{\prime} C^{\prime} B C y$ follows that $A=C^{\prime} B C$, and on a lemma 2.1 it is received: $r(Q)=r(A)=r\left(\left(C^{\prime} B\right) C\right) \leq r(C)$. As $C-$ a matrix of the size $(p \times N)$, then $r(C) \leq p$. The lemma is proved.

Using resulted above the statement, we will start the proof of independence $Q_{1}$ and $Q_{2}$. As

$$
\begin{aligned}
& Q_{0}=y^{\prime} y-\hat{\beta^{o^{\prime}}} X^{o^{\prime}} y \text {, then } \\
& y^{\prime} y=Q_{1}+Q_{2}+Q_{3}
\end{aligned}
$$

where

$$
Q_{3}=\hat{\beta}^{o^{\prime}} X^{o^{\prime}} y=y^{\prime} A_{3} y ; A_{3}=X^{o}\left(X^{o^{\prime}} X^{o}\right)^{-1} X^{o^{\prime}} .
$$

Let's define ranks of square-law forms $Q_{1}, Q_{2}$ and $Q_{3}$. As $r\left(A_{3}\right)=p_{0}$, then $r\left(Q_{3}\right)=n_{3}=p_{0} \quad[1,2,5]$. We will pass to the analysis of the square-law form

$$
Q_{2}=\sum_{l=1}^{n} \sum_{s=1}^{m_{l}}\left(y_{l s}-\bar{y}_{l}\right)^{2} .
$$

Let's enter variables $z_{l s}=y_{l s}-\bar{y}_{l}, \quad l=\overline{1, n} ; s=\overline{1, m_{l}}$. It is obvious that

$$
Q_{2}=\sum_{l=1}^{n} \sum_{s=1}^{m_{l}} z_{l s}^{2}
$$

As $\bar{y}_{l}=\frac{1}{m_{l}} \sum_{s=1}^{m_{l}} y_{l s}$, then

$$
\sum_{s=1}^{m_{l}}\left(y_{l s}-\bar{y}_{l}\right)=0 \Rightarrow \sum_{s=1}^{m_{l}} z_{l s}=0,
$$

therefore

$$
z_{l m_{l}}=-\sum_{s=1}^{m_{l}-1} z_{l s}
$$

Thus,

$$
Q_{2}=\sum_{l=1}^{n} \sum_{s=1}^{m_{l}-1} z_{l s}^{2}+\sum_{l=1}^{n} z_{l m_{l}}^{2}=\sum_{l=1}^{n} \sum_{s=1}^{m_{l}-1} z_{l s}^{2}+\sum_{l=1}^{n}\left(-\sum_{s=1}^{m_{l}-1} z_{l s}\right)^{2} .
$$

Apparently from this expression, $Q_{2}$ is the squarelaw form from $n_{2}$ variables $l=\overline{1, n} ; s=\overline{1, m_{l}-1}$, $n_{2}=\sum_{l=1}^{n}\left(m_{l}-1\right)=N-n$. As variables $z_{l s}$ are linear combinations of $y_{l s}$, and applying a lemma 5.2, we receive

$$
r\left(Q_{2}\right) \leq n_{2}=N-n .
$$

Following the similar scheme for $Q_{1}$ and applying the lemma 5 , we find

$$
r\left(Q_{1}\right) \leq n_{1}=n-p_{0} .
$$

Really, square-law form $Q_{1}$ from variables $y_{l s}$ after some transformations can be written down in a kind $Q_{1}=z^{\prime} T z, z-n$-dimensional vector, and $r(T)=n-p_{0}$.

On the basis of a consequence of a lemma 5.1 as $n_{1}+n_{2}+n_{3}=N$, we receive $r\left(Q_{1}\right)=n-p_{0}$;

$r\left(Q_{2}\right)=N-n ; r\left(Q_{3}\right)=p_{0}$.

Regarding that random variables $\frac{y_{l s}}{\sigma}, l=\overline{1, n}$, $s=\overline{1, m_{l}}$ are independent and have normal distribution $N\left(\eta_{l s}^{*}, 1\right)$, where $\eta_{l s}^{*}=\frac{\eta_{l s}}{\sigma}=\frac{\eta_{l}}{\sigma}$, then transition from equality (4.1) to equality

$$
\frac{y^{\prime} y}{\sigma^{2}}=\frac{Q_{1}}{\sigma^{2}}+\frac{Q_{2}}{\sigma^{2}}+\frac{Q_{3}}{\sigma^{2}}
$$

allows to apply the Kohran theorem. Under this theorem random variables $\frac{Q_{1}}{\sigma^{2}}, \frac{Q_{2}}{\sigma^{2}}$ and $\frac{Q_{3}}{\sigma^{2}}$ are independent and have noncentral $\chi^{2}$-distributions with $n-p_{0}$, $N-n$ and $p_{0}$ freedom degrees. Thus, independence of $Q_{1}$ and $Q_{2}$ also is proved.

Remark. Applying the Kohran theorem to calculation of parameter of non-centrality $\delta_{2}^{2}$ of the square-law form $\frac{Q_{2}}{\sigma^{2}}$, it is easy to be convinced that if the hypothesis $H_{0}$ is true or not, then $\delta_{2}^{2}=0$ i.e. the quantity $u_{2}=\frac{Q_{2}}{\sigma^{2}}$ has central $\chi^{2}$-distribution:

$$
\begin{aligned}
\delta_{2}^{2} & =\frac{1}{\sigma^{2}} \sum_{l=1}^{n} \sum_{s=1}^{m_{l}}\left(\eta_{l s}-\bar{\eta}_{l}\right)^{2}=\frac{1}{\sigma^{2}} \sum_{l=1}^{n} \sum_{s=1}^{m_{l}}\left(\eta_{l}-\frac{1}{m_{l}} \sum_{s=1}^{m_{l}} \eta_{l}\right)^{2} \\
& =\frac{1}{\sigma^{2}} \sum_{l=1}^{n} \sum_{s=1}^{m_{l}}\left(\eta_{l}-\eta_{l}\right)^{2}=0
\end{aligned}
$$

\section{References}

[1] V. S. Asaturyan, "The Theory of Planning an Experiment," Radio I Svyaz, Vol. 73, No. 3, 1983, pp. 35-241.

[2] V. A. Kolemaev, O. V. Staroverov and A. S. Turundaevski, "The Probability Theory and Mathematical Statistics," Vyishaya Shkola, Moscow, 1991, pp. 16-34.

[3] O. I. Teskin, "Statistical Processing and Planning an Experiment," MVTU, Moscow, 1982, pp. 12-26.

[4] N. I. Sidnyaev, V. A. Levin and N. E. Afonina, "Mathematical Modeling of Intensity of Heat Transmission by Means of the Theory of Planning an Experiment," 
Inzhenerno Fizicheskii Gurnal (IFG), Vol. 75, No. 2, 2002, pp. 132- 138.

[5] N. I. Sidnyaev, "The Theory of Planning Experiment and
Analysis of Statistical Data," URight, Moscow, 2011, pp. 95-220. 